\title{
In vitro synthesized immunoglobulin A from nu/ + and reconstituted nu/nu mice against a dominant surface antigen of Giardia lamblia
}

\author{
B. Gottstein ${ }^{1}$, P. Deplazes ${ }^{2}$, I. Tanner ${ }^{2}$ \\ ${ }^{1}$ Institute of Parasitology, University of Berne, P.O. Box 8466, CH-3001 Berne, Switzerland \\ 2 Institute of Parasitology, University of Zürich, Winterthurerstrasse 266a, CH-8057 Zürich, Switzerland \\ Received: 10 May 1993/ Accepted: 15 June 1993
}

\begin{abstract}
Nu} /+$ mice (ZU.ICR-strain) experimentally infected with Giardia lamblia (clone GS/M-83-H7) cleared the infection by day 45 postinfection (p.i.). Athymic nu/nu mice were reconstituted with immune Peyer's patch lymphocytes obtained from self-healed nu/ + littermates and thus acquired the potential to decrease their intestinal parasite mass. Intestinal B-cells from selfhealed $\mathrm{nu} /+$ mice as well as from immune-reconstituted athymic nude mice synthesized in vitro parasite-specific immunoglobulin A (IgA). This IgA was subsequently analyzed by immunoblotting, showing a predominant reaction with the major surface antigen (a 72000-Da polypeptide) characterizing the Giardia clone in question. The hypothesis on the causative role of intestinal IgA and immune lymphocytes in the control of G. lamblia infection thus deserves further attention.
\end{abstract}

Giardia lamblia is a protozoan parasite that resides in the small intestine of humans and other mammals. Disease manifestations vary from asymptomatic carriage to severe diarrhea and malabsorption. Although the natural history of Giardia infections is not well described, it is known that in some persons a self-cure occurs, suggesting the development of immunity or resistance, whereas others develop long-standing chronic infections. Recent studies of G. lamblia suggest that the host-parasite interactions are much more complex than previously believed. A number of studies have shown that the surface antigen profiles of Giardia are variable (Nash et al. 1983; Nash and Keister 1985; Ungar and Nash 1987) with respect to different isolates and that surface antigenic variation may occur within an isolate (Nash and Aggarwal 1986; Adam et al. 1988; Aggarwal and Nash 1988; Nash et al. 1988; Aggarwal et al. 1989). Although incompletely understood, this phenomenon is likely to play a role in facilitating chronic as well as repeated infections in humans (Gottstein et al. 1991). A complete

Correspondence to: $\mathrm{B}$. Gottstein understanding of the immunopathophysiology of infection and disease requires a model in which both the parasite and the host can be studied.

In model G. muris infections it has been shown that parasite-specific intestinal secretory immunoglobulin A (IgA) antibodies may be essential for influencing the course of infection (Anders et al. 1982; Snider and Underdown 1986; Sharma and Mayrhofer 1988; Heyworth et al. 1987, 1988; Heyworth 1989). In contrast, relatively little is known about peripheral and local immunological mechanisms involved in infections with G. lamblia. To study these problems we have recently infected neonatal mice (CR:NIH:S) with a cloned human isolate of G. lamblia (GS/M-83-H7; Gottstein et al. 1990). Followup studies analyzing the major surface antigen of the intestinal trophozoites [defined by a monoclonal antibody (MAb), G10/4] and, in parallel, the cellular and humoral immune responses showed that (a) infections in mice peaked at 2-3 weeks after inoculation and were self-cured by day 42 postinfection (p.i.), (b) the proportion of trophozoites expressing the $72000-\mathrm{Da}$ surface antigen of the initial inoculum had decreased by day 12 and approached zero by day 22 p.i., and (c) the hosts responded with a marked parasite-specific immune response (serum antibodies against the original 72000-Da surface antigen and others) and a lymphoproliferative response to G. lamblia antigens of T-lymphocytes [predominantly LY $4(\mathrm{CD} 4)^{+}$] isolated from Peyer's patches at 12 days p.i. and later. Further investigations with SCID mice and athymic nude $B A L B / c$ and respective immunocompetent control mice have subsequently revealed that a T-cell function determines self-healing, whereas the mechanism involved in inducing "parasite antigen variation" (i.e., expulsion of the initial population of the Giardia clone with the antigenic surface variant defined by mAb G10/4, subsequently replaced by new variants) appears to be independent of T-cell interactions (Gottstein and Nash 1991). The present study was designed to investigate in more detail the local immune components that may interact with the intestinal Giardia population with respect to its major surface anti- 
gen. We showed that athymic nude mice reconstituted with Peyer's patch lymphocytes from self-healed nu/ + littermates acquired the potential to synthesize intestinal IgA against the major surface Giardia antigen and that as a result of reconstitution the intestinal Giardia population was markedly reduced as compared with that in nonreconstituted control animals.

\section{Materials and methods}

\section{Experimental design}

The aim of the present study was to demonstrate the potential of intestinal murine immune cells to synthesize IgA against a single dominant surface antigen of Giardia lamblia and to provide some information on the role of parasite-specific immune lymphocytes and respective IgA in controlling infection. The experimental approach included the following:

1. Infection of nu/ + mice with $G$. lamblia to demonstrate an "antigen switch" and a self-healing course of infection. These animals were used to provide intestinal immune lymphocytes for the following experiments.

2 . In vitro synthesis by these immune lymphocytes of IgA directed against a dominant surface antigen of the Giardia clone in question; antigen specificity was assessed by enzyme-linked immunosorbent assay (ELISA) and immunoblotting.

3. Reconstitution of $\mathrm{nu} / \mathrm{nu}$ mice littermates with $\mathrm{nu} /+$ immune cells and simultaneous infection of reconstituted mice with G. lamblia; demonstration of an "antigen switch," a parasitereducing course of infection, and the synthesis in vitro of antiGiardia IgA.

\section{Animals}

The 3-day-old $\mathrm{nu} / \mathrm{nu}$ and $\mathrm{nu} /+$ mice and natural mothers used in this study were obtained as litters from the Institut für Labortierkunde, University of Zürich. The specific pathogen-free animals were of the outbred ZU.ICR strain.

\section{Parasite and experimental infections}

Five resistant (self-healing course of disease) ZU.ICR nu/ + mice and five susceptible (chronic course of disease) ZU.ICR nu/nu mice (Gottstein and Nash 1991) were infected by intragastric injection of $50000 \mathrm{G}$. lamblia trophozoites (clone GS/M-83-H7) as described previously (Gottstein et al. 1990). Five respective control animals were injected with the same volume of phosphate-buffered saline (PBS) containing 10\% TYI-S-33 medium. Animals were killed sequentially at different times (days 31 or 45 p.i., respectively). The kinetics of expression of the major surface antigen was assessed by the use of $\mathrm{mAb}$ G10/4 as described elsewhere (Gottstein et al. 1990). The origin, axenization, cultivation, and cloning of the G. lamblia GS/M-83-H7 clone (human origin) has been described by Aggarwal et al. (1989). This clone expresses a major $72-\mathrm{kDa}$ antigen on its surface, which is recognized by $\mathrm{mAb}$ G10/4. G. lamblia was cultivated in TYI-S-33 medium with antibiotics as previously described (Gottstein et al. 1990).

\section{Collection of biological samples}

Animals and organs. Lymphocyte donor $\mathrm{nu} /+$ mice (Giardia-infected mice with subsequent "self-healing" and noninfected control mice) were killed on day 45 p.i. by $\mathrm{CO}_{2}$ euthanasia. The small intestines were perfused with $10 \mathrm{ml}$ sterile PBS for removal of the intestinal contents. Peyer's patches were dissected free aseptically and placed in RPMI 1640 medium on ice prior to further processing. $\mathrm{Nu} / \mathrm{nu}$ mice (Giardia-infected mice with subsequent chronic persistence of infection and noninfected control mice) were killed on day 31 p.i. by $\mathrm{CO}_{2}$ euthanasia. The small intestines were perfused with $10 \mathrm{ml}$ sterile PBS, opened longitudinally with scissors, subsequently rinsed with sterile PBS for removal of the intestinal contents, and placed in TCM (see below) in ice prior to further processing to obtain IgA-synthesizing B-cells.

$\operatorname{Ig} A$. Intestinal cell conglomerates from reconstituted $\mathrm{nu} / \mathrm{nu}$ and nonreconstituted control mice were prepared from intestinal epithelium and lamina propria by slicing off the underlying tissue with a scalpel blade and subsequently macerating it into small pieces. This mucosal material was incubated (4 wells/mouse) in 24-well tissue-culture plates (Nunc, Roslilde, Denmark) in $1 \mathrm{ml} \mathrm{TCM}$ (see below) in a humified incubator $\left(5 \% \mathrm{CO}_{2}\right.$ and $95 \%$ air at $\left.37^{\circ} \mathrm{C}\right)$. After $24 \mathrm{~h}$ cultivation the whole cultures were frozen $\left(-20^{\circ} \mathrm{C}\right)$ and thawed (water bath, $37^{\circ} \mathrm{C}$ ) three times to disrupt the cells and the whole lysate was subsequently kept frozen at $-20^{\circ} \mathrm{C}$ until testing. For antibody-binding assays (ELISA and immunoblotting), the material was thawed and diluted $1: 2(\mathrm{v} / \mathrm{v})$ with PBSTween 20 (see below).

G. lamblia. Trophozoites were recovered by incubation of opened small intestines in ice-cold PBS (resulting in the detachment of trophozoites). The recovered $G$. lamblia trophozoites were semiquantitatively assessed for their number and directly used for immunofluorescent antibody test (IFAT) analysis as described previously (Gottstein et al. 1990).

Host immune-cell suspension. Peyer's patches from self-healed nu/ + mice were rinsed three times with ice-cold RPMI 1640 prior to isolation of single-cell suspensions. Peyer's patches were dissociated into single-cell suspensions by homogenization in Tenbroek's homogenizer. After being washed, the cells were resuspended at appropriate concentrations in tissue-culture medium [TCM:RPMI 1640 containing $10 \%$ fetal calf serum (FCS), $12 \mathrm{~m} M$ HEPES buffer, and $50 \mu M \beta$-mercaptoethanol and supplemented with $L$ glutamine ( $2 \mathrm{~m} M)$ and penicillin, streptomycin, fungizone (100 un$\mathrm{its} / \mathrm{ml}, 100 \mu \mathrm{g} / \mathrm{ml}$, and $2.5 \mu \mathrm{g} / \mathrm{ml}$, respectively)] prior to their inoculation into recipient $\mathrm{nu} / \mathrm{nu}$ mice.

\section{Reconstitution of athymic nu/nu mice}

Two athymic nude mice were reconstituted prior to Giardia infection with $5 \times 10^{6}$ lymphocytes obtained on day 45 p.i. from the Peyer's patches of (self-healed) nu/ + mice. Cell suspensions were injected intraperitoneally on the same day on which the G. lamblia infection was initiated. Nonreconstituted control animals received the same volume of TCM only.

\section{Antibody-detection assays}

Immunofluorescent antibody tests. G. lamblia trophozoites recovered from mouse small intestines were washed three times with sterile ice-cold PBS, resuspended in TYI-S-33 medium, and incubated for $1 \mathrm{~h}$ at $+37^{\circ} \mathrm{C}$ (gas phase with $7.5 \% \mathrm{CO}_{2}$ ) in Nunc flatbottom microplates. Nonadherent cells and culture medium were removed by aspiration and the adherent trophozoites were fixed for $2 \mathrm{~min}$ with 2-propanol and air-dried. Fluorescein isothiocyanate (FITC)-labeled mAb G10/4-FITC and irrelevant control mAb 6 E7-FITC were diluted 1:40 (v/v) with PBS containing $0.3 \%$ Tween 20, 10\% TYI-S-33 medium, and 0.5\% skim milk (Carnation natural nonfat dry milk) as described previously (Gottstein et al. 1991). Antibody incubation was carried out a $+37^{\circ} \mathrm{C}$ for $1 \mathrm{~h}$. The reaction was read using an inverted Olympus $\mathrm{BH}-2$ immunofluorescence microscope. 
Enzyme-linked immunosorbent assay. G. lamblia trophozoites were prepared in microplates using the same procedure described for the IFAT. We also used the same buffer solution described above to dilute TCM lysates $(1: 5, \mathrm{v} / \mathrm{v})$ and conjugate $(1: 500, \mathrm{v} / \mathrm{v})$ [antimouse $\operatorname{IgA}(\alpha$-chain-specific) conjugated to alkaline phosphatase, The Binding Site Ltd.]. Otherwise, we basically followed the procedure described earlier (Gottstein et al. 1984).

Western blotting. Resolution of G. lamblia proteins by sodium dodecyl sulfate-polyacrylamide gel electrophoresis (SDS-PAGE) and their electrophoretic transfer to Immobilon were performed as previously described (Gottstein et al. 1990) using precast 4\%-20\% gradient minigels (BioRad Co.). TCM lysates and conjugate [antimouse IgA ( $\alpha$-chain-specific) conjugated to alkaline phosphatase; the same as that described for ELISA] were diluted 1:5 and 1:500 $(\mathrm{v} / \mathrm{v})$, respectively, in the same buffer used for IFAT.

\section{Antigens}

Soluble $G$. lamblia trophozoite antigens for use in SDS-PAGE were obtained by pelleting $7 \times 10^{8}$ G. lamblia trophozoites (previously washed with sterile PBS) and resuspending them in $5 \mathrm{ml}$ PBS. Subsequent freezing $\left(1 \mathrm{~N}_{2}\right)$, thawing (water bath, $37^{\circ} \mathrm{C}$ ), and sonication $(1 \mathrm{~min}, 20 \mathrm{~W})$ provided an antigen extract after the removal of cell debris by sedimentation $(10 \mathrm{~min} ; 14000 \mathrm{~g})$.

\section{Results}

\section{Experimental infections and parasites}

The course of the Giardia lamblia GS/M-83-H7 infection in athymic nude mice (reconstituted and nonreconstituted) and in their $\mathrm{nu} /+$ littermates is shown in Table 1. The proportion of intestinal trophozoites expressing the major surface epitope binding mAb G10/4 was not explicitly listed, as in all experimental steps an antigen switch had occurred (see below). Parallel in vitro control cultures of Giardia trophozoites demonstrated the maintenance of the initial antigen variant over the experimental period (45 days).

All $\mathrm{nu} /+$ animals had either cleared their infection by day 45 p.i. or decreased the parasite burden to very few trophozoites that were detectable only after an ex- tensive microscopic search. Nonreconstituted $\mathrm{nu} / \mathrm{nu}$ mice remained heavily infected on the last respective experimental day (day 31 p.i.), whereas mice reconstituted with lymphocytes from Giardia-infected and subsequently self-healed $\mathrm{nu} /+$ mice showed a marked decrease in their parasite burden in that very few trophozoites were detectable only after an extensive microscopic search.

The analysis of G. lamblia trophozoites for the presence of the major surface epitope (72000-Da polypeptide) was assessed by IFAT and FITC-labeled $\mathrm{mAb}$ G10/ 4. A complete switch from $\mathrm{mAb}$ G10/4-positive to -negative trophozoites was in all experimental steps presented (Giardia recovered on day 14 p.i. or later).

\section{IgA synthesis as assessed by ELISA}

TCM lysates from lymphocytes cultured in vitro were assessed by ELISA for the presence of parasite-specific IgA; the respective results are shown in Table 1. All experimentally infected $\mathrm{nu} /+$ animals had primed B-cells in that these were subsequently capable of synthesizing parasite-specific $\operatorname{IgA}$ in vitro, in contrast to nonreconstituted nu/nu mice. The two mice reconstituted with lymphocytes from Giardia-infected and subsequently selfhealed nu/ + mice exhibited Giardia-specific IgA-binding activity in ELISA. Controls, including noninfected nu/ + and infected but nonreconstituted nu/nu mice, were negative, i.e., they showed no antibody-binding activity in the parasite-specific IgA ELISA.

\section{$\operatorname{Ig} A$ synthesis as assessed by immunoblotting}

Immunoblotting was used to assess the Giardia molecules that were responsible for IgA-binding activity in the experiments described above. The migration site of the 72000-Da major surface antigen characterizing our G. lamblia clone (GS/M-83-H7) was visualized with an $\mathrm{mAb}$ G10/4-alkaline phosphatase conjugate. Under the present experimental conditions, parasite-specific intesti-
Table 1. Analysis of Giardia lamblia trophozoites recovered from experimentally infected neonatal mice and demonstration of a respective humoral IgA response by ELISA using in vitro cultivated intestinal lymphocyte lysates

\begin{tabular}{|c|c|c|c|c|}
\hline $\begin{array}{l}\text { Number } \\
\text { of mice }\end{array}$ & Strain & $\begin{array}{l}\text { Experimentally } \\
\text { infected/ } \\
\text { reconstituted }\end{array}$ & $\begin{array}{l}\text { Giardia } \\
\text { recovered } \\
\text { after necropsy }^{\mathrm{a}}\end{array}$ & $\begin{array}{l}\text { IgA detected in cultured } \\
\text { intestinal lymphocyte lysates } \\
\text { by ELISA, mean (range) }^{\mathrm{b}}\end{array}$ \\
\hline 5 & $\mathrm{Nu} / \mathrm{nu}$ & $\mathrm{No} / \mathrm{no}$ & $(5 \times)$ & $0.04(0.01-0.05)$ \\
\hline 5 & $\mathrm{Nu} / \mathrm{nu}$ & Yes/no & $+++(5 x)$ & $0.05(0.01-0.07)$ \\
\hline 5 & $\mathrm{Nu} /+$ & No/no & $-\quad(5 \times)$ & $0.04(0.02-0.05)$ \\
\hline 3 & $\mathrm{Nu} /+$ & Yes/no & $(+)(3 \times)$ & $0.12(0.11-0.13)$ \\
\hline 2 & $\mathrm{Nu} /+$ & Yes/no & $-\quad(2 \times)$ & $0.26(0.12-0.41)$ \\
\hline 2 & $\mathrm{Nu} / \mathrm{nu}$ & Yes/yes & $(+)(2 \times)$ & $0.13(0.12-0.13)$ \\
\hline
\end{tabular}

${ }^{a}$ Giardia recovered on day 31 p.i. All trophozoites had demonstrated a change in their surface antigen variant as shown by a lack of binding capacity with mAb G10/4; the intensity of infection was estimated by microscopic analysis of the fluid obtained after perfusion and of the small intestines and was arbitrarily and semiquantitatively classified as follows: +++ , heavy parasite burden; $(+)$, a few trophozoites detectable only in the whole intestine.

${ }^{\mathrm{b}}$ Data in boldface indicate positive results based upon a threshold of $\mathrm{A}_{404 \mathrm{~nm}}=0.10$ as determined by the mean value +3 SD for the noninfected controls 


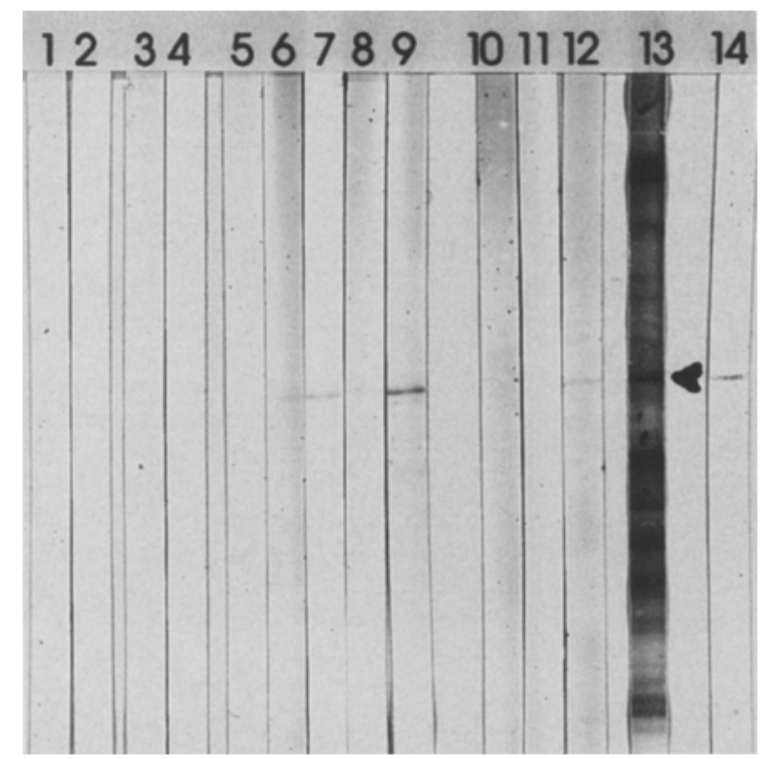

Fig. 1. Immunoblot analysis of parasite-specific in vitro synthesized IgA using antigens from the initial Giardia lamblia clone GS/M-83H7 and lymphocytes from some selected and representative animals. Lane 1: $\mathrm{Nu} / \mathrm{nu}$, no infection/no reconstitution; lane $2: \mathrm{nu} / \mathrm{nu}$, Giardia-infected, no reconstitution; lanes 3, 4: nu/ + , no infection; lanes 5 9: $\mathrm{nu} /+$, Giardia-infected; lane 10: $\mathrm{nu} / \mathrm{nu}$, Giardia-infected, no reconstituion; lanes 11, 12: nu/nu, Giardia-infected, reconstituted with immune cells. Identification of the site of the 72000-Da major surface antigen (arrow) was done by molecularweight markers (not shown) and by a reference-strip (lane 14) reaction with $\mathrm{mAb}$ G10/4. Lane 13: Protein stain with India ink

nal $\operatorname{IgA}$ bound only to the 72000 -Da major surface antigen (Fig. 1), in contrast to total serum immunoglobulins from similar previous experiments (Gottstein et al. 1990), which had recognized other additional bands. The binding activity to the 72000-Da major surface antigen was restricted to experimentally infected $\mathrm{nu} /+$ mice and to one of two $\mathrm{nu} / \mathrm{nu}$ mice reconstituted with lymphocytes from Giardia-infected and subsequently self-healed $\mathrm{nu} /+$ mice.

\section{Discussion}

Our previous investigations had demonstrated that a lymphocyte proliferative response to Giardia-antigen stimulation was limited to Peyer's patch cells and could not be detected in peripheral lymph nodes or spleen cells. The use of immunodeficient mice in previous studies provided a number of interesting findings. In the $\mathrm{nu} / \mathrm{nu}$ and $\mathrm{nu} /+$ mouse experiment, it became evident that the course of the $G$. lamblia infection was mainly mediated by functional thymus-dependent T-lymphocytes. These lymphocytes and potentially related synthesized antibodies obviously allowed the corresponding host animals to clear the infection, whereas a basic lack of the respective functional lymphocytes resulted in the chronic persistence of a vast parasite population in the small intestine (Gottstein and Nash 1991). These circumstances indicate the development of protective immunity in $(\mathrm{nu} /+)$ mice against the homologous G. lamblia clone under investigation and the failure of $(\mathrm{nu} / \mathrm{nu})$ mice to develop such immune protection.

The results of the present series of experiments further support the idea that antibody responses, especially with respect to the local synthesis of parasite-specific secretory IgA, may be of causative relevance in vivo. Although $\mathrm{nu} / \mathrm{nu}$ mice demonstrated the capacity to mount a parasite-specific serum Ig response following experimental infection, they lacked the potential to synthesize intestinal IgA against the $72000-\mathrm{Da}$ major surface antigen characterizing our Giardia clone. Nu/nu mice reconstituted with immune Peyer's patch cells obtained from self-healed $\mathrm{nu} /+$ mice acquired the potential to synthesize intestinal IgA against the 72000-Da major surface antigen and simultaneously acquired the potential to decrease their intestinal parasite mass. These results represent the first demonstration of parasite-specific IgA synthesis by immune B-cells from mice experimentally infected with $G$. lamblia, implying their potential relevance for the elimination of $G$. lamblia trophozoites. Of course, we do not exclude the possibility that other types of immune responses not demonstrated in the present work may also be causatively involved in modulating the loss of the initial 72-kDa epitope and the subsequent course of infection. For further experiments, we plan to dissect the nature of the immune reactions listed above by reconstituting SCID mice with subsets of immune cells provided by reconstituted mouse intestines as described in this paper and to prove the in vitro cytotoxicity of anti-72-kDa-IgA as previously shown with $\mathrm{mAb}$ G10/4 (Nash and Aggarwal 1986).

The present results and findings emphasize the role of intestinal immune lymphocytes and IgA in recognizing Giardia surface antigens with respect to protective mechanisms.

Acknowledgements. We would like to express our thanks to Thomas Bruderer for technical assistance. This work was supported by a grant provided by the Schweizerische Stiftung für Medizinisch-Biologische Stipendien.

\section{References}

Adam RD, Aggarwal A, Lal AA, De La Cruz VF, McCutchan T, Nash TE (1988) Antigenic variation of a cysteine-rich protein in Giardia lamblia. J Exp Med 167:109-118

Aggarwal A, Nash TE (1988) Antigenic variation of Giardia lamblia in vivo. Infect Immun 56:1420-1423

Aggarwal A, Merritt JW, Nash TE (1989) Cysteine-rich variant surface proteins of Giardia lamblia. Mol Biochem Parasitol $32: 39-48$

Anders RF, Robert-Thomson IC, Mitchell GF (1982) Giardiasis in mice: analysis of humroal and cellular immune response to Giardia muris. Parasite Immunol 4:47-57

Gottstein B, Nash TE (1991) Antigenic variation in Giardia lamblia: infection of congenitally athymic nude and scid mice. Parasite Immunol 13:649-659

Gottstein B, Eckert J, Woodtli W (1984) Determination of parasitespecific immunoglobulins using the ELISA in patients with echinococcosis treated with mebendazole. Parasitol Res $70: 385-389$ 
Gottstein B, Harriman GR, Conrad JT, Nash TE (1990) Antigenic variation in Giardia lamblia: cellular and humoral immune response in a mouse model. Parasite Immunol 12:659-673

Gottstein B, Stocks NI, Shearer GM, Nash TE (1991) Human cellular immune response to Giardia lamblia. Infection 19:421426

Heyworth MF (1989) Intestinal IgA responses to Giardia muris in mice depleted of helper $T$ lymphocytes and in immunocompetent mice. J Parasitol 75:246

Heyworth MF, Carlson JR, Ermak TH (1987) Clearance of Giardia muris infection requires helper/inducer T lymphocytes. J Exp Med 165:1743-1748

Heyworth MF, Kung JE, Caplin AB (1988) Enzyme-linked immunosorbent assay for Giardia lamblia-specific $\operatorname{IgA}$ in mouse intestinal secretions. Parasite Immunol 10:713-717

Nash TE, Aggarwal A (1986) Cytotoxicity of monoclonal antibodies to a subset of Giardia isolates. J Immunol 136:2628-2632
Nash TE, Keister DB (1985) Differences in excretory-secretory products and surface antigens among 19 isolates of Giardia. J Infect Dis 152:1166-1171

Nash TE, Gillin FD, Smith PD (1983) Excretory-secretory products of Giardia lamblia. J Immunol 131:2004-2010

Nash TE, Aggarwal A, Adam RD, Conrad JT, Merritt JW (1988) Antigenic variation in Giardia lamblia. J Immunol 141:636-641

Sharma AW, Mayrhofer G (1988) A comparative study of infections with rodent isolates of Giardia duodenalis in inbred strains of rats and mice and in hypothymic nude rats. Parasite Immunol 10:169-179

Snider DP, Underdown BJ (1986) Quantitative and temporal analyses of murine antibody response in serum and gut secretions to infection with Giardia muris. Infect Immun 52:271-278

Ungar BLP, Nash TE (1987) Cross-reactivity among different Giardia lamblia isolates using immunofluorescent antibody and enzyme immunoassay techniques. Am J Trop Med Hyg 37:283289 